\title{
DEEP GRAPH REGULARIZED LEARNING FOR BINARY CLASSIFICATION
}

\author{
Minxiang Ye $\quad$ Vladimir Stankovic ${ }^{\star} \quad$ Lina Stankovic ${ }^{\star} \quad$ Gene Cheung ${ }^{\dagger}$ \\ * Department of Electronic \& Electrical Engineering, University of Strathclyde, Glasgow, UK \\ ${ }^{\dagger}$ Department of Electrical Engineering \& Computer Science, York University, Toronto, Canada
}

\begin{abstract}
With growing interest in data-driven classification, deep learning is now prevalent due to its ability to learn feature mapping functions solely from data. For very small training sets, however, deep learning, even with traditional regularization techniques, often overfits, resulting in sub-par classification performance. In this paper, we propose a novel binary classifier deep learning method, based on an iterative quadratic programming (QP) formulation with a graph Laplacian regularizer (GLR), combining the merits of model-based and data-driven approaches. Specifically, the proposed network employs a convolutional neural network (CNN) to learn deep features, which are used to define edge weights for a graph to pose a convex QP problem. Further, we design a novel loss function to penalize samples at the class boundary during semi-supervised learning. Results demonstrate that, given a small-size training dataset, our network outperforms several state-of-the-art classifiers, including CNN, model-based GLR and dynamic graph CNN classifiers.
\end{abstract}

Index Terms - graph Laplacian regularization, binary classification, semi-supervised learning, deep learning

\section{INTRODUCTION}

Given a sufficient amount of representative training data, data-driven classifier learning based on deep neural networks (DNN) provides state-of-the-art classification performance for various large-scale learning tasks [1], [2]. However, in many practical cases, collecting labeled data is either overly expensive or time-consuming. One example is medical image analysis [3], where only a limited number of images are annotated by experts, due to the tedious and costly labelling process.

Given insufficient training data, robust classifier learning remains a challenge. Data-driven techniques, like convolutional neural networks $(\mathrm{CNN})$ and recurrent neural networks $(\mathrm{RNN})$ enable powerful feature extraction, but tend to overfit given limited labeled data. One remedy is regularization. In the literature, five regularization types are investigated [4]: (1) regularization via data transformation to augment the training dataset, e.g., in image classification tasks [5], [6], data augmentations are performed on the training data by combining different affine transformations and color transformations; (2) regularization via networks by performing different sampling and weight-sharing methods on the mapping functions within the neural networks, such as dropout [7], skip-connections [8], [9], etc; (3) regularization via regularization term that is independent of the targets, such as adding weight decay or smoothness prior, method of [1], etc; (4) regularization via optimization by adopting warm-start, different update methods and termination methods, as reviewed in [10]; and (5) regularization via the error function by departing from classic, mean squared error or cross-entropy loss functions, to better reflect data distribution by minimizing systematic empirical risk, such as applying Dice coefficient optimization [11] to achieve robustness to class imbalance.

In an orthogonal development, recent advance in graph signal processing (GSP) [12] provides spectral tools to analyze and process signals on graphs. Specifically, binary classifiers can be interpreted as piecewise smooth signals on graphs [13], restored using a graphsignal smoothness prior [14]. For example, in [15] negative edge weights are introduced into the graph to achieve more robust classification. Given a set of features, [16] propose an alternating graphbased binary classifier for interpolating missing labels by learning relative weights of the features.

Motivated by the above, to address the problem of classifier learning with small labeled data, we propose a novel neural network based on iterative quadratic programming (QP) with graph Laplacian regularizer (GLR), combining the merits of model-based and data-driven approaches. Specifically, we use a CNN to learn deep features organically, which are used to define a notion of "distance" to compute edge weights in a sparse graph. The graph is used to specify the GLR, resulting in a convex QP problem with numerical stability guarantee [17]. Further, we design a modified loss function that reflects the quality of learned underlying graph used for GLR, promoting connections between the nodes with the same labels, and penalizing nodes at the class boundary during training to improve generalization. Through extensive experiments, given small labeled data, we show that our proposed network outperforms several state-of-the-art binary classification methods, including support vector machine (SVM) [18], GLR-based approaches [13], classic CNN-based classifier, and more recent, deep metric based $\mathrm{k}$ nearest neighbor (KNN) [19] and dynamic graph CNN classifier [20].

\section{RELATION TO PRIOR WORK}

Deep learning has achieved success in various machine learning tasks that involve natural images, video, speech and other data with an underlying Euclidean structure [21]. More recently, with growing interest in non-Euclidean data, deep learning is used to learn the geometric structure in various tasks, e.g., node classification in graph structured data [22], image denoising of point cloud data tasks [17], point cloud classification and segmentation [20].

In particular, tackling the problem of graph learning for nonEuclidean data, [23] propose spectral convolutional layer that assigns degrees of freedom for each eigenvector of the graph Laplacian. Spectrum-free methods [22], [24], [25] adopt polynomial of the graph Laplacian as features into CNNs to perform classification given a fixed irregular graph structure [21]. [26] employ an edge convolutional layer to learn spatial features from neighboring nodes given a fixed skeleton graph. [20] introduce an iterative KNN graph reconstruction process based on learned features from multiple edge convolutional layers. The results demonstrate the capability of edge convolution operation in terms of feature generalization for classifi- 
cation tasks for non-Euclidean data, such as point-cloud data.

GLR [27] has been used for data classification, e.g., [13] propose a supervised classification method by minimizing total variation on graph where nodes of the graph are indexed by observed classification labels, forming a graph signal, and are connected by positive edge weights that reflect similarity between observed features. More recently, by introducing the negative edge weights into the graph, [15] show sufficient robustness of classification to noise in the labels for small-scale datasets. Focusing on semi-supervised binary classification, [16] jointly optimize underlying graph construction and the classifier graph-signal restoration. [17] propose a deep-image denoising framework that couples a fully-differentiable graph Laplacian regularization layer with a lightweight CNN for pre-filtering and learning 8-connected pixel adjacency graph structures based on the learned features of CNN. The results demonstrate that given a small dataset, the network outperforms CNN-based approaches by avoiding overfitting and achieves comparable results when sufficient data is available.

Unlike the regression task considered in [17], we employ the GLR as a classifier using a partially labelled dataset for training. By integrating deep metric learning and GLR within CNN, we propose a novel deep graph regularized neural network that simultaneously learns deep features and the graph structure to perform GLR.

\section{DEEP GRAPH-BASED LEARNING}

In this section, we present our proposed deep graph regularized neural network, for semi-supervised learning when the amount of labeled data available to train the model is very small. The main idea is to iteratively use $\mathrm{CNN}$ to learn the deep features and the optimal underlying graph for graph Laplacian regularization.

We start by defining the problem and introducing notation, closely following related work [13], [15]-[17], [19]. Then, we present the steps of our method, and finally show the proposed network architecture used to implement the proposed method.

\subsection{Problem Formulation and Notation}

Let $\mathbf{X}=\left\{x_{1}, \ldots, x_{N}\right\}$ be a set of samples or data instances to be classified, where each instance $x_{i}$ is a vector of observed features. Let $\mathbf{Y}=\left\{y_{1}, \ldots, y_{N}\right\}$ be a set of corresponding binary labels, most of them unknown. Given $\mathbf{X}=\{x\}$ and $\mathbf{Y}=\{y\}$, the binary classifier should learn, during training, an approximate function $F(x)$ that maps each input instance $x$ to a label $y$, which is then used to determine the labels of new, testing samples. This is accomplished by presenting to the classifier numerous examples of $(x, y)$ pairs that constitute the training set. Typically, for machine learning, the size of the training set is at least equal to the size of the testing set. The problem addressed in this paper is the case when the size of the training set is much smaller than that of the testing set.

Let $\mathbf{Y}=\left\{\dot{\mathbf{Y}}=\{-1,1\}^{M}, \mathbf{0}^{N-M}\right\}$ be a set of labelled observations, where we set to zero all $N-M$ unknown labels (to be estimated during testing), with $N \gg M$, and $\dot{\mathbf{Y}}=\{-1,1\}^{M}$ is a set of known labels that correspond to instances $\left\{x_{1}, \ldots, x_{M}\right\}$ used for training.

We construct a graph $\mathbf{G}=(\mathbf{X}, \mathbf{E}, \mathbf{W})$, where each instance, $x_{i}$, corresponds to a node $i$, in $\mathbf{G} . \mathbf{E}=\left\{e_{i, j}\right\}, i, j \in\{1, \ldots, N\}$, denotes a set of graph edges, with $e_{i, j}=1$ if there is an edge between nodes $i$ and $j$, and otherwise $e_{i, j}=0$, and $\mathbf{W}$ is the matrix of the corresponding edge weights $w_{i, j}$. The graph Laplacian matrix is given by $\mathbf{L}=\mathbf{D}-\mathbf{A}$, where $\mathbf{A}$ is a symmetric adjacency matrix with each entry $a_{i, j}=a_{j, i}=\max \left(w_{i, j} \cdot e_{i, j}, w_{j, i} \cdot e_{j, i}\right)$, and $\mathbf{D}$ is a degree matrix with entries $d_{i, i}=\sum_{j=1}^{N} a_{i, j}$, and $d_{i, j}=0$ for $i \neq j$. Note that $e_{i, j}$ and $w_{i, j}$ are problem specific, and have been the subject of optimization, for example, in [16].

Our first task is to learn $e_{i, j}$ and $w_{i, j}$. Once we know $e_{i, j}$ and $w_{i, j}$, we can employ graph Laplacian regularization, similarly to [15]-[17], to regularize $\mathrm{CNN}$ used to learn deep features. In addition, we estimate the quality of the learned graph, expressed as edge and weight loss, and feed this loss back to the Optimization module of CNN to drop training samples that potentially sit at the class boundary and would worsen generalization.

\subsection{Deep Metric Learning}

Let $\mathcal{F}(x)$ be a feature representation function that projects the original feature space of $x$ into a deep metric feature space [19]. That is, first, for each training sample $x_{i}$, we use CNN to find the optimal 'deep' features $\mathcal{F}\left(x_{i}\right)$, without regularization.

Next, we design a graph $\mathbf{G}$, using $\mathcal{F}\left(x_{i}\right)$ as samples and corresponding labels $\dot{\mathbf{Y}}$ as graph signal. To learn the optimal set of edges and edge weights of the graph, we need a cost function that promotes connecting nodes that correspond to the same labels and penalizes connections between the nodes with opposite labels, while constraining the graph to be sparse and connected, needed for efficient graph Laplacian regularization.

It has been shown that triplet loss-based deep networks achieve better classification accuracy than conventional DNNs for image classification, person re-identification and face recognition, as triplet loss effectively learns a deep metric to better represent the distance between any two instances [19], [28], [29]. Using triplet loss, we formulate the loss function of graph edge E's connectivity learning as:

$\operatorname{Loss}_{\mathbf{E}}=\sum_{a, p, n}^{N}\left[\alpha_{\mathbf{E}}-\left\|\mathcal{F}\left(x_{a}\right)-\mathcal{F}\left(x_{n}\right)\right\|_{2}^{2}+\left\|\mathcal{F}\left(x_{a}\right)-\mathcal{F}\left(x_{p}\right)\right\|_{2}^{2}\right]_{+}$,

where $a$ and $p$ are the nodes with the same label, i.e., $y_{a}=y_{p} \in \dot{\mathbf{Y}}$, and node $n$ is with the opposite label, i.e., $y_{a} \neq y_{n} \in \dot{\mathbf{Y}}$. Operator $[\cdot]_{+}$is a $\operatorname{ReLu}$ activation function which is equivalent to $\max (\cdot, 0)$. The loss function (1) promotes small/large Euclidean distance between the nodes with the same/opposite labels, while keeping minimum margin $\alpha_{\mathbf{E}}$ between these two Euclidean distances. The loss value of error function-based regularization term (1) will be fed to ADAM optimizer for updating CNN to find improved deep features, $\mathcal{F}^{*}\left(x_{i}\right)$, that take into account graph construction.

As in [15], [17], in order to obtain a sparse connected graph, we introduce a constraint that the graph must be a KNN graph and define a graph edge $e_{i, j}$ between the $i$-th and $j$-th node as:

$$
e_{i, j}= \begin{cases}1, & \text { if } x_{i} \in B_{\gamma}\left(x_{j}\right) \text { or } x_{j} \in B_{\gamma}\left(x_{i}\right) \\ 0, & \text { otherwise }\end{cases}
$$

where $B_{\gamma}\left(x_{j}\right)$ is the $\gamma$ nearest nodes to node $j$, based on distance $\left\|\mathcal{F}^{*}\left(x_{i}\right)-\mathcal{F}^{*}\left(x_{j}\right)\right\|_{2}^{2}$, used to control the sparsity of graph connectivity (see Sec. 3.2 in [30]). We iteratively optimize (1) to learn $\mathcal{F}^{*}(\cdot)$ by back propagation via ADAM Optimizer. We then adopt (2) to find the optimal degree of the nodes $\gamma$ by decreasing $\gamma$ from a fully connected graph, based on the classification accuracy measured on the validation set.

Note that, given insufficient data, deep metric function $\mathcal{F}^{*}$ would quickly overfit, calling for extra regularization that would penalize samples that sit at the class boundaries and could potentially otherwise lead to poor model generalization. Typically, in the deep learning framework, a combination of $l_{1}$ or $l_{2}$ regularization, dropout, and/or batch normalization are performed as a part of the loss function [31]. Instead, we hypothesize and show in Section 4 
that these techniques are ineffective when the training set is very small, and therefore employ graph Laplacian regularization to add extra regularization to the loss function. Given $\gamma$ found via (2), first, we assign edge weight $\left\{w_{i, j}\right\}$ by:

$$
w_{i, j}=\exp \left(-\frac{\left\|\mathcal{F}_{r}\left(x_{i}\right)-\mathcal{F}_{r}\left(x_{j}\right)\right\|_{2}^{2}}{2 \sigma^{2}}\right),
$$

where Gaussian kernel function is used to limit edge weights within an appropriate range. $\mathcal{F}_{r}(x)$ denote regularized deep features obtained via a $\mathrm{CNN}$. Once $\mathbf{W}$ is found, we define weight loss as:

$$
\begin{aligned}
\operatorname{Loss}_{\mathbf{W}}= & \sum_{a, p, n}^{M}\left[\alpha_{\mathbf{W}}-\left\|\mathcal{F}_{r}\left(x_{a}\right)-\mathcal{F}_{r}\left(x_{n}\right)\right\|_{2}^{2} \cdot \pi_{a, n}\right. \\
& \left.+\left\|\mathcal{F}_{r}\left(x_{a}\right)-\mathcal{F}_{r}\left(x_{p}\right)\right\|_{2}^{2} \cdot \pi_{a, p}\right]_{+} \\
\Pi= & \left\{\pi_{i, j}\right\}=\Theta(\ddot{\mathbf{Y}}, \dot{\mathbf{Y}}),
\end{aligned}
$$

where $\Theta$ is the activation function (see (6)) that estimates how much attention should be given to each edge, and $\ddot{\mathrm{Y}}$ is a solution of

$$
\operatorname{argmin}_{\mathbf{U}}\left\{\|\mathbf{U}-\dot{\mathbf{Y}}\|_{2}^{2}+\mu \mathbf{U L} \mathbf{U}^{T}\right\} \text {. }
$$

Note that (5) is the graph Laplacian regularization step that attempts to find the smoothest graph signal, $\mathbf{U}$, for a given graph, that is close to the observed set of labels, $\dot{\mathbf{Y}} . \pi_{a, p}$ and $\pi_{a, n}$ are the amount of attention, i.e., edge loss weights, given to edge nodes with the same and opposite labels, respectively. To guarantee that the solution $\ddot{Y}$ to the quadratic programming $(\mathrm{QP})$ problem (5) is numerically stable, we adopt Theorem 1 from [17] by setting an appropriate conditional number $\kappa$. The resulting smoothness prior factor $\mu$ is then calculated as: $\mu=(\kappa-1) /\left(2 d_{\max }\right)$, where $d_{\max }$ is the maximum degree of the vertices in graph $\mathbf{G}$.

The obtained loss, $\operatorname{Loss}_{\mathbf{W}}$, is fed back to the CNN for regularization. By calculating iteratively (3), (5), (4), batch-by-batch, and feeding back the loss to CNN to update $\mathcal{F}_{r}$ the loss of graph edge weight is minimized based on the edges with high attention value, while learning the optimal regularized deep metric function $\mathcal{F}_{r}$.

\subsection{Implementation: Proposed Network Architecture}

To solve the above deep metric learning problem we propose a deep graph regularized network (DGR-Net), shown in Fig. 1, comprising two sub-networks: (I) KNN-Net (deep metric learning-based KNN classifier): used to estimate the optimal $\gamma$, see (2), where an undirected KNN graph is constructed by learning a deep metric function through minimization of $\operatorname{Loss}_{\mathbf{E}}$ (1). (II) Graph-Net (graph-based binary classifier): used to learn edge weights $\mathbf{W}$ via (3) and (4) to construct a weighted undirected graph, $\mathbf{G}$, constrained by the optimal $\gamma$, to perform graph Laplacian regularization to obtain the solution $\ddot{\mathbf{Y}}$ (5). To guarantee the solution $\ddot{\mathrm{Y}}$ to (5) is numerically stable, we heuristically set conditional number, $\kappa$, as defined in [17], to 50 in all our experiments.

The proposed CNNs are shown in Fig. 2, which differ only in the loss function used: $\mathrm{CNN}_{\mathcal{F}}$ within $\mathrm{KNN}-\mathrm{Net}$ uses edge loss (1), while $\mathrm{CNN}_{\mathcal{F}_{r}}$ weight loss (3), both trained via ADAM optimizer with initial learning rate of 0.002 linearly decreasing with the number of epochs. We use the same distance margin of 10 for both $\alpha_{\mathbf{E}}$ and $\alpha_{\mathbf{W}}$ in $(1,3)$. For each epochs, we use batch size of 6 , each batch comprising 120 nodes, thus we randomly sample $6 \cdot 120$ samples into the network. This results in 6 graphs to regularize the training.

Note that to achieve sparse, connected graph, we disconnect relatively low weighted edges that are most likely connecting incorrectly labeled nodes. We adopt $\mathrm{KNN}$-graph construction based on (2), where optimal maximum number of neighbors $\gamma$ is obtained via grid-search by evaluating classification error of an KNN-classifier on the validation set. The resulting $\gamma$ is then used in the GR-Net

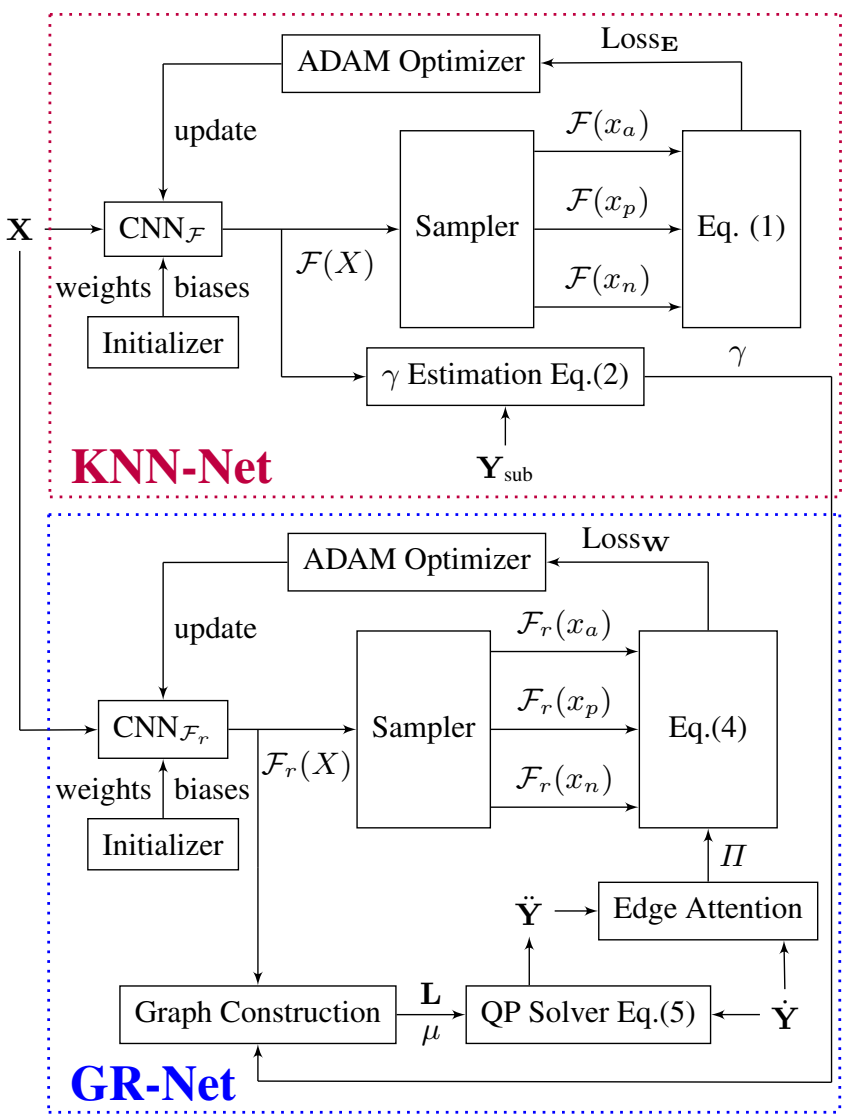

Fig. 1: Overall block diagram of the proposed DGR-Net. KNN-Net first learns a deep metric function $\operatorname{CNN}_{\mathcal{F}}$ by minimizing $\operatorname{Loss}_{\mathbf{E}}$. We adopt a Sampler to extract triplets: $\mathcal{F}\left(x_{a}\right)$ - random instances in $\mathbf{X}$, $\mathcal{F}\left(x_{p}\right)$ - random instance subsets of $\mathbf{X}$ with the same label as $\mathcal{F}\left(x_{a}\right)$, $\mathcal{F}\left(x_{n}\right)$ - random instance subsets of $\mathbf{X}$ with the opposite label to $\mathcal{F}\left(X_{a}\right)$. Once we obtain $\mathcal{F}^{*}(\cdot)$, we construct KNN graphs using (2) given a subset of training data $\left(\mathbf{X}_{\text {sub }} \mid \mathbf{Y}_{\text {sub }}\right)$ not used for minimizing Loss $_{\mathbf{E}}$, i.e., $\mathbf{X}_{\text {sub }} \subset \mathbf{X}$ and $\mathbf{Y}_{\text {sub }} \subset \dot{\mathbf{Y}}$. An optimal $\gamma$ is then estimated by evaluating KNN classification accuracy on those KNN graphs where random subsets of nodes are manually unlabeled. $\gamma$ is then used as maximum degree of undirected $\mathrm{KNN}$-graph constructed in the following GR-Net by minimizing Lossw. GR-Net learns a regularized deep metric function $\mathrm{CNN}_{\mathcal{F}_{r}}$ to assign edge weights and constructs undirected KNN graphs constrained by $\gamma$. Then, it uses GLR to estimate $\ddot{\mathbf{Y}}$ given $\dot{\mathbf{Y}}$ via (5). Attention is then computed by an activation function to penalize Loss $\mathbf{W}$.

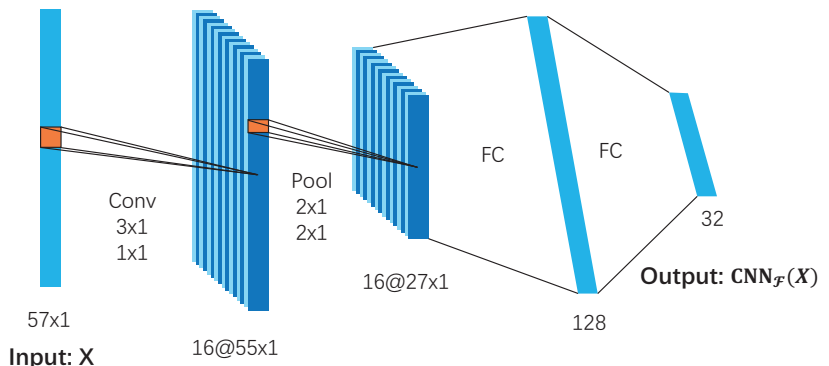

Fig. 2: $\mathrm{CNN}_{\mathcal{F}}$ and $\mathrm{CNN}_{\mathcal{F}_{r}}$ neural network. FC denotes fully connected layer. 
for pruning edge weights $\mathbf{W}$ during graph weighting. Like in [15], [17], we adopt Gaussian kernel as normalization function $P$ in (3) for assigning edge weights $\mathbf{W}$ of graph $\mathbf{G}$. Once $\mathbf{W}$ is defined, $\mathbf{E}$ is computed as a mask matrix for undirected $\mathrm{KNN}$ graph, where only top $\gamma$ entries $\left\{e_{i, j_{1}}, \ldots, e_{i, j_{\gamma}}\right\}$ of the $i$-th node are set to 1 .

As mentioned in Sec. 3.2, we then solve the MAP problem (5), passing graph Laplacian $\mathbf{L}$ and partial labeled observations $\dot{\mathbf{Y}}$. In case of insufficient training data, edge attention activation $\Theta$, implemented as a nonzero thresholded step as in (6), will be used to dropout some edges with relatively large changes between $\ddot{\mathbf{Y}}$ and original $\dot{\mathbf{Y}}$ after applying graph Laplacian regularization. This facilitates to penalize learning on edges with relative low confidence (outliers). Therefore, the overall training regularizes $\mathrm{CNN}_{\mathcal{F}_{r}}$ better than typically dropout random neural units in the network.

$$
\Theta(\ddot{\mathbf{Y}}, \dot{\mathbf{Y}})= \begin{cases}1, & \text { if }|\ddot{\mathbf{Y}}-\dot{\mathbf{Y}}|>\varepsilon \\ 0, & \text { if }|\ddot{\mathbf{Y}}-\dot{\mathbf{Y}}| \leq \varepsilon\end{cases}
$$

where threshold $\varepsilon$ is used to measure how confident a node's label is and also helps to control the sparsity of edge attention matrix $\Pi$. Under the assumption of ineffective graph Laplacian regularization during the early training, we adopt a decayed $\varepsilon$ decreasing linearly from 2 to 0.6 during training to ensure that we start regularizing $\mathrm{CNN}_{\mathcal{F}_{r}}$ until we obtain acceptable Laplacian matrix $\mathbf{L}$ without influence the earlier training.

\section{RESULTS}

In this section, we evaluate our proposed network benchmarked against different classifiers using classification error rate as performance metric. We select two binary-class datasets from Knowledge Extraction based on Evolutionary Learning dataset (KEEL) [32]: (1) Phoneme: classification of nasal (class 0 ) and oral sounds (class 1), consisting of 5404 instances (frames) described by 5 phonemes of digitized speech. (2) Spambase: determining whether an email is spam (class 0) or not (class 1), with 4597 email messages summarized by 57 particular words or characters.

We compare the proposed DGC network against the following classifiers: (1) linear SVM (2) SVM with radial basis function kernel (denoted by SVM-RBF) (3) a purely graph-based classifier with smoothness prior (Graph) (4) a classical CNN, consisting of one $\mathrm{CNN}$ with two fully connected layers at the end; $\mathrm{CNN}$ block has a convolutional layer, a max pooling layer, and one dropout layer before each fully connected layer; $l_{2}$ regularization is applied to all trainable layers (denoted by $\mathrm{CNN}$ ) (5) a graph-based $\mathrm{CNN}$ with multiple KNN graph construction blocks, which also learns the optimal graph structure as the proposed method but using edge convolution (denoted by DynGraph-CNN) [20]; batch normalization, $l_{2}$ regularization and dropout are all used (6) a KNN classifier using deep metric learning [19] (DML-KNN) with $l_{2}$ regularization and dropout.

We conduct our experiments by randomly splitting each dataset into training, validation and testing sets. We select 10, 15, 20, 25, $30 \%$ of instances as training sets, $10 \%$, instances as validation set, and the remaining instances contribute towards the testing set. Classification error rates are measured by running 20 experiments for each dataset. We use the same random seed setting across all classification methods and remove all duplicated instances to ensure a fair comparison. Hyper-parameters used for each experiment are obtained from the validation set by grid search.

As shown in Tables $1 \& 2$, given insufficient amount of data, DGC consistently outperforms the other 6 benchmarks, as observed by lower classification error rates. One reason is that all other neural network-based classifiers overfit to the insufficient training data. Indeed, the proposed method uniformly outperforms all benchmarks
Table 1: Classification Error Rate (\%) for the Phoneme Dataset for various sizes of the training dataset expressed as the proportion of the total dataset $(\%)$.

\begin{tabular}{|c|c|c|c|c|c|}
\hline Proportion (\%) & 10 & 15 & 20 & 25 & 30 \\
\hline SVM-Linear & 25.73 & 25.79 & 25.62 & 25.63 & 25.60 \\
\hline SVM-RBF & 20.81 & 20.32 & 19.78 & 19.45 & 19.05 \\
\hline Graph & 23.09 & 22.92 & 22.72 & 22.34 & 22.17 \\
\hline CNN & 20.69 & 20.22 & 19.51 & 19.12 & 18.91 \\
\hline DynGraph-CNN & 22.12 & 20.20 & 19.39 & 19.21 & 18.40 \\
\hline DML-KNN & 20.37 & 19.44 & 19.31 & 19.18 & 18.12 \\
\hline DGC & $\mathbf{1 9 . 8 6}$ & $\mathbf{1 9 . 3 7}$ & $\mathbf{1 8 . 9 3}$ & $\mathbf{1 8 . 7 8}$ & $\mathbf{1 7 . 8 9}$ \\
\hline
\end{tabular}

Table 2: Classification Error Rate (\%) for the Spambase Dataset for various sizes of the training dataset expressed as the proportion of the total dataset $(\%)$.

\begin{tabular}{|c|c|c|c|c|c|}
\hline Proportion (\%) & 10 & 15 & 20 & 25 & 30 \\
\hline SVM-Linear & 10.26 & 9.83 & 9.52 & 9.06 & 8.94 \\
\hline SVM-RBF & 10.04 & 9.30 & 9.00 & 8.61 & 8.41 \\
\hline Graph & 20.22 & 20.10 & 19.68 & 19.13 & 18.72 \\
\hline CNN & 9.72 & 9.18 & 8.75 & 8.65 & 8.26 \\
\hline DynGraph-CNN & 11.84 & 10.71 & 9.52 & 9.38 & 9.09 \\
\hline DML-KNN & 9.20 & 8.26 & 7.97 & 7.73 & 7.44 \\
\hline DGC & $\mathbf{9 . 0 8}$ & $\mathbf{8 . 1 8}$ & $\mathbf{7 . 6 4}$ & $\mathbf{7 . 5 2}$ & $\mathbf{7 . 3 8}$ \\
\hline
\end{tabular}

for the training dataset sizes between $10 \%-30 \%$ of the total dataset.

The Graph method, based on graph Laplacian regularization, does not learn features, resulting in the worst performance among all tested schemes. DynGraph-CNN network [20] performs worse than $\mathrm{CNN}$ when the amount of training data is very low, since the network is deeper than the CNN benchmark, closing the performance gap as the training dataset size increases. DML-KNN is always the second best method indicating that learning deep metric via triplet loss provides performance gain. Further improvements of the proposed DGC over DML-KNN are due to the effectiveness of the proposed graph Laplacian regularization.

\section{CONCLUSIONS}

A deep graph regularized network architecture for binary classifier learning for the case of insufficient training data is proposed and benchmarked against classical and state-of-the-art classifiers. The proposed solution embeds graph Laplacian regularization, a proven method to combat insufficient training dataset, into a CNN architecture used for deep feature learning via new formulation of a loss function that takes into account the quality of the underlying graph used to calculate the graph Laplacian. The proposed network consistently outperforms six benchmarks, including a prior graph Laplacian regularization approach, conventional CNN and SVM classifiers, as well as latest state-of-the-art dynamic graph $\mathrm{CNN}$ architecture that uses geometric learning to find optimal graph structure.

Future work will comprise extending the work to handle noise in the training dataset. Another direction is extending the work to the multi-class classification problem.

\section{ACKNOWLEDGMENT}

This project has received funding from the European Union's Horizon 2020 research and innovation programme under the Marie Skłodowska-Curie grant agreement No 734331. The University of Strathclyde gratefully acknowledges the support of NVIDIA Corporation with the donation of the Titan Xp GPU used for this research. 


\section{References}

[1] A. Krizhevsky, I. Sutskever, and G. E. Hinton, "Imagenet classification with deep convolutional neural networks," in Advances in neural information processing systems, 2012, pp. 1097-1105.

[2] A. Graves, A.-R. Mohamed, and G. Hinton, "Speech recognition with deep recurrent neural networks," in ICASSP 2013, 2013, pp. 6645-6649.

[3] Y. Xu, T. Mo, Q. Feng, P. Zhong, M. Lai, and E. I. Chang, "Deep learning of feature representation with multiple instance learning for medical image analysis," in ICASSP2014, May 2014, pp. 1626-1630.

[4] J. Kukačka, V. Golkov, and D. Cremers, "Regularization for deep learning: A taxonomy," ArXiv:1710.10686, 2017.

[5] L. Perez and J. Wang, "The effectiveness of data augmentation in image classification using deep learning," ArXiv:1712.04621, 2017.

[6] A. Mikołajczyk and M. Grochowski, "Data augmentation for improving deep learning in image classification problem," in 2018 IIPhDW, May 2018, pp. 117-122.

[7] G. E. Hinton, N. Srivastava, A. Krizhevsky, I. Sutskever, and R. R. Salakhutdinov, "Improving neural networks by preventing co-adaptation of feature detectors," ArXiv:1207.0580, 2012.

[8] K. He, X. Zhang, S. Ren, and J. Sun, "Deep residual learning for image recognition," in Pro.e IEEE Conf. Computer Vision and Pattern Recognition, 2016, pp. 770-778.

[9] G. Huang, Z. Liu, L. Van Der Maaten, and K. Q. Weinberger, "Densely connected convolutional networks.," in CVPR, vol. 1, 2017, p. 3.

[10] A. C. Wilson, R. Roelofs, M. Stern, N. Srebro, and B. Recht, "The marginal value of adaptive gradient methods in machine learning," in Advances in Neural Information Processing Systems, 2017, pp. 4148-4158.

[11] F. Milletari, N. Navab, and S.-A. Ahmadi, "V-net: Fully convolutional neural networks for volumetric medical image segmentation," in 2016 Fourth Intl. Conf. 3D Vision (3DV), IEEE, 2016, pp. 565-571.

[12] A. Ortega, P. Frossard, J. Kovacevic, J. M. F. Moura, and P. Vandergheynst, "Graph signal processing: Overview, challenges, and applications," in Proceedings of the IEEE, vol. 106, no.5, May 2018, pp. 808-828.

[13] A. Sandryhaila and J. M. F. Moura, "Classification via regularization on graphs," in 2013 IEEE Global Conference on Signal and Information Processing, Dec. 2013, pp. 495-498.

[14] J. Pang and G. Cheung, "Graph Laplacian regularization for image denoising: Analysis in the continuous domain," IEEE Transactions on Image Processing, vol. 26, no. 4, pp. 17701785, 2017.

[15] G. Cheung, W. Su, Y. Mao, and C. Lin, "Robust semisupervised graph classifier learning with negative edge weights," IEEE Transactions on Signal and Information Processing over Networks, vol. 4, no. 4, pp. 712-726, Dec. 2018.

[16] C. Yang, G. Cheung, and V. Stankovic, "Alternating binary classifier and graph learning from partial labels," in AsiaPacific Signal and Information Processing Association Annual Summit and Conference 2018, Nov. 2018.
[17] J. Zeng, J. Pang, W. Sun, and G. Cheung, "Deep graph Laplacian regularization for robust denoising of real images," ArXiv 1807.11637, 2018.

[18] C. Cortes and V. Vapnik, "Support-vector networks," Machine learning, vol. 20, no. 3, pp. 273-297, 1995.

[19] E. Hoffer and N. Ailon, "Deep metric learning using triplet network," in International Workshop on Similarity-Based Pattern Recognition, Springer, 2015, pp. 84-92.

[20] Y. Wang, Y. Sun, Z. Liu, S. E. Sarma, M. M. Bronstein, and J. M. Solomon, "Dynamic graph CNN for learning on point clouds," ICLR 2017, vol. abs/1801.07829, 2018.

[21] M. M. Bronstein, J. Bruna, Y. LeCun, A. Szlam, and P. Vandergheynst, "Geometric deep learning: Going beyond euclidean data," IEEE Signal Processing Magazine, vol. 34, no. 4, pp. 18-42, Jul. 2017.

[22] T. N. Kipf and M. Welling, "Semi-supervised classification with graph convolutional networks," ArXiv:1609.02907, 2016.

[23] F. Scarselli, M. Gori, A. C. Tsoi, M. Hagenbuchner, and G. Monfardini, "The graph neural network model," IEEE Transactions on Neural Networks, vol. 20, no. 1, pp. 61-80, Jan. 2009.

[24] J. Bruna, W. Zaremba, A. Szlam, and Y. LeCun, "Spectral networks and locally connected networks on graphs," ArXiv:1312.6203, 2013.

[25] M. Defferrard, X. Bresson, and P. Vandergheynst, "Convolutional neural networks on graphs with fast localized spectral filtering," in Advances in Neural Information Processing Systems 29, Curran Associates, Inc., 2016, pp. 3844-3852.

[26] X. Zhang, C. Xu, and D. Tao, "Graph edge convolutional neural networks for skeleton based action recognition," ArXiv:1805.06184, 2018.

[27] D. I. Shuman, S. K. Narang, P. Frossard, A. Ortega, and P. Vandergheynst, "The emerging field of signal processing on graphs: Extending high-dimensional data analysis to networks and other irregular domains," IEEE Signal Processing Magazine, vol. 30, no. 3, pp. 83-98, May 2013.

[28] F. Schroff, D. Kalenichenko, and J. Philbin, "Facenet: A unified embedding for face recognition and clustering," in Proceedings of the IEEE Conf. Computer Vision and Pattern Recognition, 2015, pp. 815-823.

[29] A. Hermans, L. Beyer, and B. Leibe, "In defense of the triplet loss for person re-identification," ArXiv:1703.07737, 2017.

[30] G. L. Miller, S.-H. Teng, W. Thurston, and S. A. Vavasis, "Separators for sphere-packings and nearest neighbor graphs," Journal of the ACM, vol. 44, no. 1, pp. 1-29, 1997.

[31] P. Lemberger, "On generalization and regularization in deep learning," ArXiv:1704.01312, 2017.

[32] J. Alcala-Fdez, A. Fernández, J. Luengo, J. Derrac, S. Garc'ia, L. Sanchez, and F. Herrera, "Keel data-mining software tool: Data set repository, integration of algorithms and experimental analysis framework," Journal of MultipleValued Logic and Soft Computing, vol. 17, pp. 255-287, Jan. 2010. 